\title{
Tofacitinib Induced Psoriasiform Lesion in a Patient With Rheumatoid Arthritis
}

\author{
Kemal Erol'i(D), Ragıp Ertaş ${ }^{2}$ \\ ${ }^{1}$ Department of Physical Medicine and Rehabilitation, Division of Rheumatology, Kayseri City Hospital, Kayseri, Turkey \\ ${ }^{2}$ Department of Dermatology, Kayseri City Hospital, Kayseri, Turkey
}

\begin{abstract}
Paradoxical psoriasis or psoriasiform lesion is an adverse effect, represented by the occurrence of a psoriasiform lesion or exacerbation of psoriasis caused by the drugs normally used for the management of psoriasis. In this article, we present the first case of a 45 -year-old male patient with rheumatoid arthritis who developed psoriasiform lesions following treatment with tofacitinib, and highlight the possible pathogenetic mechanisms involved in such an occurrence.

Keywords: Paradoxical, psoriasis, rheumatoid arthritis, tofacitinib.
\end{abstract}

Paradoxical psoriasis or psoriasiform $(\mathrm{PPsO})$ lesion is an adverse effect, represented by the occurrence of a psoriasiform lesion or exacerbation of psoriasis caused by the drugs normally used for the management of psoriasis. Tofacitinib, a small molecule that inhibits janus kinases (JAKs), is a drug used for the treatment of psoriasis and also rheumatoid arthritis (RA). ${ }^{1,2}$ Herein, to the best of our knowledge, we present the first case of a male patient with RA who developed psoriasiform lesions following treatment with tofacitinib, and discuss the possible pathogenetic mechanisms.

\section{CASE REPORT}

A 45-year-old male patient with RA was referred for newly onset skin rashes on his arms and legs after starting the treatment with tofacitinib (Figure 1). He was taking methotrexate
$5 \mathrm{mg} /$ weekly, folic acid $5 \mathrm{mg} /$ weekly and tofacitinib $5 \mathrm{mg}$ twice a day for RA. Disease had started nine years ago, first with pain and swallowing on his ankle. Within one year, arthritis developed on wrist, metacarpophalangeal (MCP) and proximal interphalangeal (PIP) joints, with no involvement in distal interphalangeal (DIP) joints. The patient had no inflammatory back pain symptoms. Psoriasis was not present in his or his family's medical history. Laboratory tests were negative for antinuclear antibody, extractable nuclear antigen profile, anti-cyclic citrullinated peptide, rheumatoid factor and human leukocyte antigen B27. Erythrocyte sedimentation rate was $26 \mathrm{~mm} /$ hour and C-reactive protein was $12.6 \mathrm{mg} / \mathrm{L}(0-5 \mathrm{mg} / \mathrm{L})$. There were bilateral ulnar styloid erosions, carpal erosions and osteopenia on his hand X-ray; marginal erosions on fifth $\mathrm{MCP}$, second MCP, third PIP joint, subluxation on second $\mathrm{MCP}$ joint on right hand; joint erosion

Received: April 05, 2019 Accepted: July 12, 2019 Published online: October 11, 2019

Correspondence: Kemal Erol, MD. Kayseri Şehir Hastanesi Romatoloji Kliniği, 38080 Kocasinan, Kayseri, Turkey. Tel: +90352 - 3157700 e-mail: erolk.md@gmail.com 


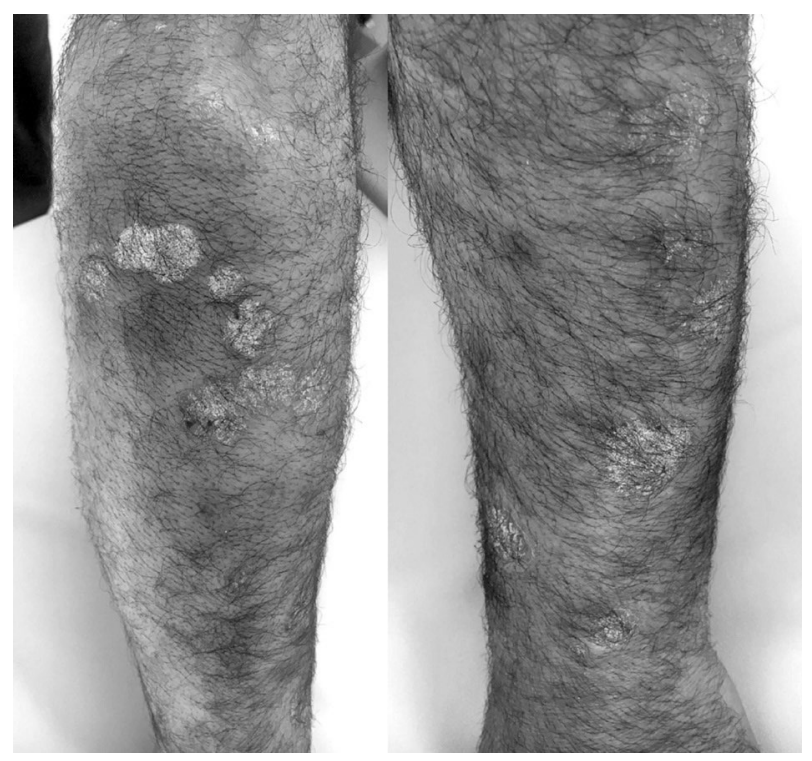

Figure 1. Lesions on patient's arms.

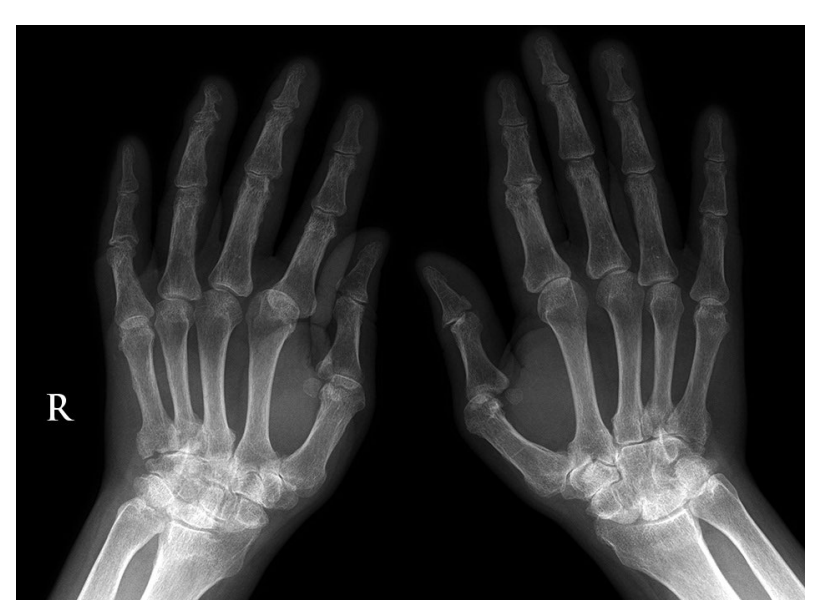

Figure 2. Patient's hand X-ray.

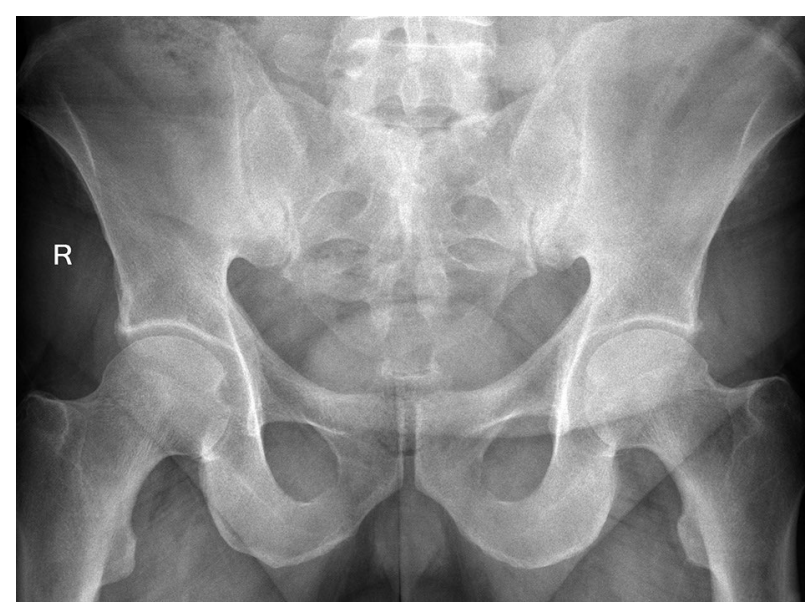

Figure 3. Pelvis anteroposterior X-ray. and subluxation of first MCP, erosion and joint space narrowing of second PIP joint on left hand (Figure 2). DIP joints were normal on both hands except two osteophytes. On his pelvis anteroposterior X-ray, sacroiliac joint spaces were normal (Figure 3). Patient's type of joint involvement and radiological findings were consistent with RA but not psoriatic arthritis. In this course, he had used methotrexate, sulfasalazine, leflunomide, hydroxychloroquine and glucocorticoids at different times. Because of the ineffectiveness of the conventional synthetic disease-modifying anti-rheumatic drugs, etanercept was started. Then, etanercept induced skin rashes with unknown characteristics and was stopped subsequently. After improving of the skin rashes, rituximab was started but discontinued due to the ineffectiveness. Tofacitinib was started when his disease activity was high (disease activity score-28 [DAS28] >5.1). Skin rashes appeared on the third month of the tofacitinib therapy while disease activity was low (DAS28=2.8). We consulted the dermatology. Dermatological examination revealed multiple, clearly demarcated, silvery, squamous and erythematous papules and plaques on the arms, legs, and ankles. Skin biopsy was performed. Epidermal parakeratosis, hyperkeratosis, focal hypogranulosis, and acanthosis with elongation of the rete ridges were observed in the skin biopsy. He was diagnosed with psoriasis. We decided that tofacitinib induced psoriasiform lesions, so tofacitinib was discontinued and leflunomide was started. After two months, lesions were improved. A written informed consent was obtained from the patient.

\section{DISCUSSION}

To the best of our knowledge, this is the first case of tofacitinib induced PPsO lesion. It is well known that tumor necrosis factor inhibitors (TNFi's) are used for the treatment of inflammatory diseases including psoriasis while they may also cause PPsO. ${ }^{3}$ However, there is no data about the association of PPsO and tofacitinib which has been used for RA since 2012 and which is relatively new for the treatment of psoriasis. Tofacitinib is a smallmolecule, inhibiting JAK1 and JAK3 and, to a lesser extent, JAK2. ${ }^{4}$ In psoriasis pathogenesis, 
many of the cytokines take role through the JAK-signal transducers and activators of transcription (STAT) signaling pathway like the expression of interleukin (IL)-23 and IL-23 dependent releasing of IL-17, differentiation of T helper type 1 (Th1) cells. In RA pathogenesis, production of IL-17 and interferon-gamma (IFN- $\gamma$ ) and proliferation of CD4 T cells (presumably Th1 and Th17) is dependent to JAK-STAT signaling pathway. ${ }^{5,6}$ Therefore, tofacitinib is an effective agent both in the treatment of psoriasis and RA. Moreover, a great number of case series reported the effectiveness of tofacitinib in various inflammatory diseases. ${ }^{7,8}$

Tumor necrosis factor-alpha (TNF- $\alpha$ ) inhibits plasmacytoid dendritic cells (pDCs) and IFN- $\alpha$ production which is the key pathogenetic event in early psoriasis. Therefore, it has been proposed that TNFi agents, inhibiting TNF- $\alpha$, may favor an overproduction of IFN- $\alpha$ by pDCs that may explain the underlying mechanism of TNFi induced PPsO. ${ }^{3}$ Tofacitinib inhibits JAKs and causes downstream of multiple cytokines including IFN- $\alpha$, thus in the pathogenesis of tofacitinib induced $\mathrm{PPsO}$, imbalance of IFN- $\alpha$ and IFN- $\gamma$ may play a role. ${ }^{9}$ Another possible pathogenetic mechanism of tofacitinib induced $\mathrm{PPsO}$ like the TNFi induced $\mathrm{PPsO}$ is the expansion of pathogenic Th1 and Th17 cells in the peripheral lymph nodes due to the decreased Th1 and Th17 cells in the inflamed synovium in RA. ${ }^{10}$

In conclusion, therapeutic agents like tofacitinib and TNFi, which affect through the specific immune pathways in the management of the immune-mediated inflammatory disorders, are potential causes of the paradoxical inflammatory disorders like $\mathrm{PPsO}$. This is a severe, but fortunately rare problem for clinicians administering these drugs. It is important to pay attention to the reports of further cases in various biologic or targeted drugs used in various inflammatory diseases and associated with paradoxical inflammatory disorders and to try to understand the underlying mechanisms that may enable the management of these disorders.

\section{Declaration of conflicting interests}

The authors declared no conflicts of interest with respect to the authorship and/or publication of this article.

\section{Funding}

The authors received no financial support for the research and/or authorship of this article.

\section{REFERENCES}

1. Azevedo A, Torres T. Tofacitinib: A New Oral Therapy for Psoriasis. Clin Drug Investig 2018;38:101-12.

2. Smolen JS, Landewé R, Bijlsma J, Burmester G, Chatzidionysiou K, Dougados $\mathrm{M}$, et al. EULAR recommendations for the management of rheumatoid arthritis with synthetic and biological diseasemodifying antirheumatic drugs: 2016 update. Ann Rheum Dis 2017;76:960-77.

3. Fiorino G, Danese S, Pariente B, Allez M. Paradoxical immune-mediated inflammation in inflammatory bowel disease patients receiving anti-TNF- $\alpha$ agents. Autoimmun Rev 2014;13:15-9.

4. Kawalec P, Sladowska K, Malinowska-Lipien I, Brzostek T, Kózka M. European perspective on the management of rheumatoid arthritis: clinical utility of tofacitinib. Ther Clin Risk Manag 2017;14:1529.

5. Cinats A, Heck E, Robertson L. Janus kinase inhibitors: a review of their emerging applications in dermatology. Skin Therapy Lett 2018;23:5-9.

6. Tanaka Y, Maeshima K, Yamaoka K. In vitro and in vivo analysis of a JAK inhibitor in rheumatoid arthritis. Ann Rheum Dis 2012;71:70-4.

7. Gök K, Cengiz G, Erol K, Ozgocmen S. Tofacitinib suppresses disease activity and febrile attacks in a patient with coexisting rheumatoid arthritis and familial Mediterranean fever. Acta Reumatol Port 2017;42:88-90.

8. Rimar D, Alpert A, Starosvetsky E, Rosner I, Slobodin G, Rozenbaum M, et al. Tofacitinib for polyarteritis nodosa: a tailored therapy. Ann Rheum Dis 2016;75:2214-6.

9. Pollard KM, Cauvi DM, Toomey CB, Morris KV, Kono $\mathrm{DH}$. Interferon- $\gamma$ and systemic autoimmunity. Discov Med 2013;16:123-31.

10. Notley CA, Inglis JJ, Alzabin S, McCann FE, McNamee KE, Williams RO. Blockade of tumor necrosis factor in collagen-induced arthritis reveals a novel immunoregulatory pathway for Th1 and Th17 cells. J Exp Med 2008;205:2491-7. 